\title{
Role of Fluctuation-Induced Interactions in the Axial Segregation of Granular Materials
}

\author{
I. Zuriguel, J. F. Boudet, Y. Amarouchene, and H. Kellay \\ Centre de Physique Moleculaire Optique et Hertzienne, UMR 5978, \\ U. Bordeauxl 351 cours de la Liberation, 33405 Talence cedex France
}

(Received 11 May 2005; published 16 December 2005)

\begin{abstract}
The movement of a few large diameter spheres immersed in a granular medium composed of smaller beads in a rotating cylinder is studied. We evidence attractions and repulsions between the large spheres depending on the rotation frequency. The large spheres also show relative position fluctuations which are Gaussian. A complete study of this problem sheds new light on the problem of size segregation in granular materials and points to the importance of fluctuation-induced interactions.
\end{abstract}

Size segregation in granular materials is an intriguing phenomenon that poses many fundamental physics questions [1,2]. The issues raised cross the frontiers between statistical mechanics, nonlinear physics, and out of equilibrium physics $[3,4]$. Much industrial processes also suffer from or use such segregation. Segregation occurs in natural granular flows such as avalanches and land slides giving rise to a change of landscape. There are different situations where segregation by size occurs. One of these situations, studied for over 70 years, is known to occur in cylindrical tumblers [5]. Here, a long cylinder is filled halfway with grains of two different sizes and is rotated slowly around the cylinder long axis. After a few rotations, axial segregation starts and the two species separate in bands that are equally spaced: regions consisting mostly of the smaller grains are intercalated by regions containing the larger diameter grains. Such an instability has been widely studied both experimentally and theoretically [6-9]. A difference in the repose angles of the two species against the surface of the cylinder has been invoked as a possible mechanism for such axial segregation [8]. Other mechanisms such as depletion $[10,11]$ have been invoked recently for horizontally and vertically vibrated two-dimensional layers. For three-dimensional granular layers subjected to vertical vibration three mechanisms have been identified in a recent experiment: convection, inertia, and buoyancy [12].

Here we present results that shed new light on this phenomenon and point to the relevance of induced particle interactions for axial segregation. We use a long cylinder that is half filled with small glass beads to which we have added a few steel spheres of much larger diameter. The steel spheres are sufficiently heavy to be almost completely covered by the small grains and remain at a fixed distance from the surface during the course of the experiment. Our main observation is that at high rotation speeds the larger spheres are equally spaced from each other as seen in Fig. 1: the assembly of large spheres shows long range order along the cylinder long axis. This situation is stable for very long times. Upon reducing the rotation speed below a threshold frequency, these spheres start to form aggregates: couples, triplets, and higher order aggregates can be obtained as seen in Fig. 1. By studying what happens for just two spheres we show that they experience either a mutual attraction below the threshold frequency or a mutual repulsion above this threshold. An essential feature of the induced interactions is the fluctuation of the small beads.

The experimental apparatus consists of a long cylinder of $19 \mathrm{~cm}$ in length with a diameter of $1.8 \mathrm{~cm}$. This cylinder was attached with a gasket to a continuous motor delivering stable rotation frequencies from 0.1 to $10 \mathrm{~Hz}$. The cylinder was half filled with small diameter glass beads $(0.05 \mathrm{~cm})$ and the larger diameter $(0.6 \mathrm{~cm})$ steel spheres. Video imaging, both at a standard rate (25 images per second) and at faster rates (typically 250 images per second), was used to track the movement of the steel spheres. The cylinder was usually illuminated with a broad white light and images were taken in transmission so the steel spheres appeared black in a white background. A homemade particle tracking program was used to extract the coordinates of the steel spheres versus time.

What are the mechanisms leading to the observed aggregation and what is its kinetics? To answer these questions we studied what happens for just two spheres. In a typical experiment, we fill the cylinder to a controlled

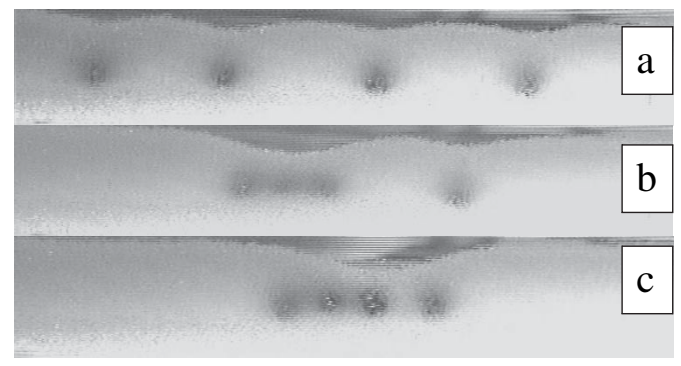

FIG. 1. Photographs of the particle configurations in a cylindrical cell half filled with small glass beads of $0.05 \mathrm{~cm}$ in diameter including 4 steel spheres of $0.6 \mathrm{~cm}$ in diameter for two different frequencies. (a) Above the threshold frequency, the large spheres (the dark spots) are equidistantly separated from each other. (b) and (c) Below threshold, aggregation occurs showing a triplet and the formation of a quadruplet. 
height and bring the two steel spheres as close together as possible. For each height we determine the frequency, $f_{\text {th }}$, at which the two spheres separate from each other. At rotation frequencies $f$ greater than $f_{\text {th }}$, the two spheres separate while they stay together as an aggregate for $f$ smaller than $f_{\text {th }}$. The threshold frequency increases as the filling ratio increases but seems to saturate for large height while for small heights it was difficult to stabilize the cluster. The height of filling was varied between $40 \%$ and $60 \%$ of the tube length. Figure 2 shows this transition quantitatively and depicts the mean separation distance $R_{\text {mean }}$ between the two spheres as a function of the rotation frequency for a filling height ratio of $44 \%$. The mean separation starts out small at low frequencies, and is close to the particle diameter $(0.6 \mathrm{~cm})$, and increases continuously to nearly two particle diameters as the frequency increases towards $f_{\text {th }}$. Above $f_{\text {th }}$, the cluster is unstable and the two spheres drift apart from each other to reach a new stationary position between 3 and $4 \mathrm{~cm}$. We have also tracked the fluctuations of the separation $R$. A typical probability density function (PDF) of the relative position fluctuations, for a fixed frequency below $f_{\text {th }}$, is shown in the left inset to Fig. 2. This PDF is Gaussian for all the frequencies we examined $\left(f<f_{\text {th }}\right)$. However, the width and the mean position increase as the frequency increases as shown in Fig. 2 where the right inset depicts the variation of the width of the PDFs versus the frequency. The transition from a cluster to separate spheres seems gradual at first, reminiscent of a second order transition. A large jump in $R_{\text {mean }}$ is then observed as $f_{\text {th }}$ is crossed. The existence of this threshold was also tested using other diameters for the steel spheres $(0.3$ and $0.5 \mathrm{~cm})$ for which we found an increase of $f_{\text {th }}$ ( 1 and $1.6 \mathrm{~Hz}$, respectively)

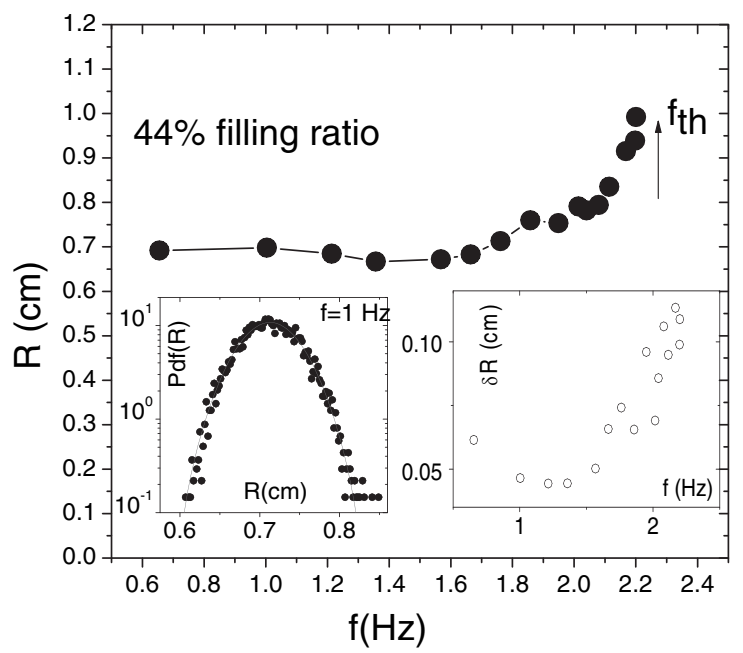

FIG. 2. The mean separation $R_{\text {mean }}$ of a cluster of two steel spheres vs the frequency. Above $f_{\text {th }}, R_{\text {mean }}$ jumps to values around $3 \mathrm{~cm}$. Left inset: PDF of the relative position fluctuations between the two spheres. The solid line is a Gaussian fit. The width of the PDFs of the fluctuations in $R$ vs the frequency is shown in the right inset. versus the diameter. We have also used $0.75 \mathrm{~cm}\left(f_{\text {th }}=\right.$ $5 \mathrm{~Hz}$ for $44 \%$ filling) glass spheres with roughly similar results. Lower density spheres (teflon spheres) have a tendency to undergo a rotation around an axis parallel to the cylinder long axis and to segregate vertically so they were not used for this study.

Why is this transition observed? In order to examine this issue we measured the velocity fluctuations of the small particles. Visual observations indicated that the particles in the region between the two steel spheres moved slower and fluctuated less than outside this region. These measurements use laser Doppler velocimetry to track the velocity and its rapid fluctuations, of the small particles at the surface. These measurements were carried out versus $f$ in the region between the steel spheres and outside this region. The results are shown in Fig. 3. The quantity plotted is $\delta U^{2}=-\left[U_{\text {rms }}^{2}\right.$ (out) $+V_{\text {rms }}^{2}$ (out) $-U_{\text {rms }}^{2}($ in $)-$ $\left.V_{\mathrm{rms}}^{2}(\mathrm{in})\right]$ versus the frequency $f$. Here $U$ is the component of the velocity in the flow direction and $V$ is the component of the velocity transverse to the flow. The regions between the steel spheres and outside the two spheres are noted (in) and (out), respectively. The velocity fluctuations are quantified via $U_{\text {rms }}=\sqrt{\left\langle\left(U-U_{\text {mean }}\right)^{2}\right\rangle}$ and $V_{\text {rms }}=\sqrt{\left\langle V^{2}\right\rangle}$. This figure shows that the fluctuations in the outside region are larger than between the two spheres. The fluctuations then become similar at a threshold frequency very similar to the one determined in Fig. 2. Above this frequency they remain roughly the same. A possible explanation is that the proximity of the two spheres confines the small particles and reduces their fluctuations. That the difference in the velocity fluctuations vanishes at the threshold and above it indicates that these fluctuations contribute in an important way to the attraction observed. The "granular temperature $T$," as measured via the square of the rms value of the velocity fluctuations $[13,14]$, and therefore the pressure, is different in the two regions. As the frequency increases, this temperature difference decreases and vanishes beyond $f_{\text {th }}$. The small particles in the outside region are therefore exerting an inward pressure [the pressure difference between the two regions is $\left.\delta P=\frac{1}{2} \rho \delta U^{2}[13,14]\right]$ on the two

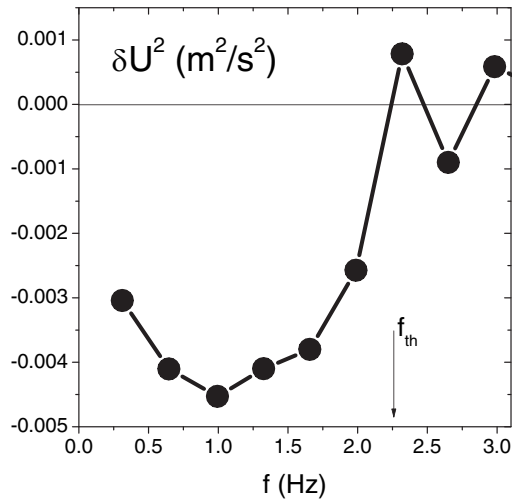

FIG. 3. The difference in the velocity fluctuations in the region between the two spheres and outside the two spheres vs the driving frequency. Above $f_{\text {th }}$ this difference is zero. 
spheres forcing them to cluster together. Once the fluctuations are balanced on either side, the flow of the small particles into the region between the spheres becomes important, pushing them apart from each other and giving rise to the separated state.

In a second step, we studied the dynamic aspects of this problem. For the attraction, the two spheres were first separated and the rotation started afterwards. For the repulsion, the two spheres were set as close as possible to each other and the frequency set to the desired one above $f_{\text {th }}$. Figures 4(a) and 4(b) show the evolution of the relative distance $R$ between the two spheres versus time for two frequencies: below $f_{\text {th }}$ where $R$ is a decreasing function of elapsed time and above $f_{\text {th }}$ where $R$ is an increasing function of time. These graphs show that both the attraction and the repulsion are long range. The range of the attraction increases with decreasing frequency and becomes as large as the half tube length. For the repulsion, the maximal separation can be as large as $4 \mathrm{~cm}$. In both cases, we could fit the relative separation, $R(t)$, using three forces per unit mass. The first one is written in the form $A / R$, with the coefficient $A$ being positive or negative for repulsion or attraction. The choice of this functional form is dictated by the fact that the repulsion and the attraction are long ranged and second of all by simplicity. The second one is of the form $-B v$, where $v$ is the instantaneous relative velocity of the two spheres, and which is similar to a Stokes drag acting to slow the movement of the spheres. For the third one, we used a constant $C$. The presence of this term is necessary to obtain a well-defined final position for the repulsion as we will see below. This term may account for a constant drag independent of the velocity as evidenced by recent experiments [15-17] or most probably a dynamic

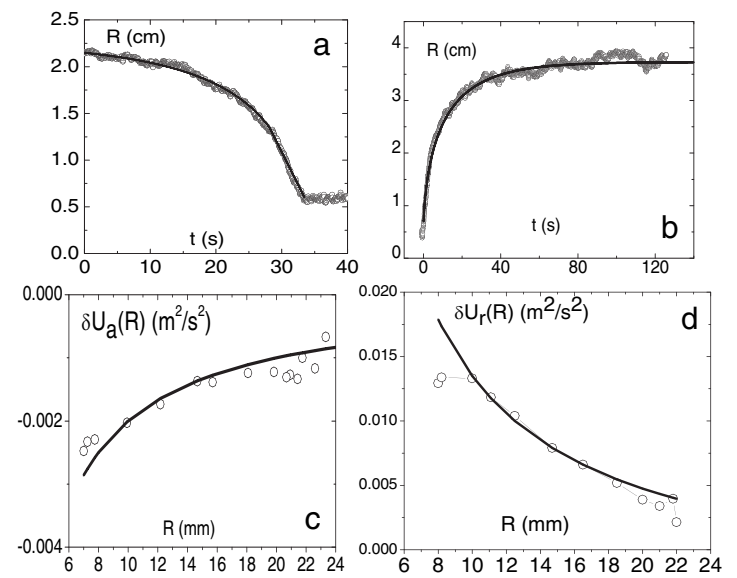

FIG. 4. (a) Approach of two steel spheres for a frequency below $f_{\text {th }}, f=0.95 \mathrm{~Hz}$ at $42 \%$ filling. (b) Relative distance $R$ between the two spheres vs time for a frequency above $f_{\text {th }}, f=$ $3.3 \mathrm{~Hz}$ at $40 \%$ filling. The solid lines are fits using the expression given in the text. (c) $\delta U_{a}^{2}(R)$ vs $R$ for $f=0.8 \mathrm{~Hz}<f_{\text {th }}$ and $44 \%$ filling height. (d) $\delta U_{r}^{2}(R)$ vs $R$ for $f=3 \mathrm{~Hz}>f_{\text {th }}$ and $44 \%$ filling height. The solid lines in (c) and (d) show a $1 / R$ dependence. friction coefficient. The three terms used together can be numerically integrated to obtain the variation of $R$ versus time. Fits to the data are superimposed on the data points in these figures. The agreement is very good for both the repulsion and the attraction. What is the meaning of the three forces at play, what is their origin, and what fixes the values of the three parameters $A, B$, and $C$ ?

The attraction, as argued above, is due to differences in temperature and therefore in pressure between the outside region and the inner region. These fluctuations give rise to an entropic force proportional to $\delta U_{a}^{2}(R)=-\left[U_{\mathrm{rms}}^{2}\right.$ (out) + $V_{\mathrm{rms}}^{2}($ out $\left.)-U_{\mathrm{rms}}^{2}(R)-V_{\mathrm{rms}}^{2}(R)\right]$ which is plotted versus $R$ in Fig. 4(c). The measurements of the velocity and its fluctuations in the inner region and of the position $R$ were carried out simultaneously while the two spheres drifted towards each other. This force is negative and decreases in absolute value as $R$ increases in agreement with our supposed dependence in $1 / R$. The continuous curve in Fig. 4(c) shows the $1 / R$ dependence; the agreement is satisfactory.

For the repulsion, we have also measured the velocity and its fluctuations in the inner region and the separation $R$ simultaneously while the two spheres drifted apart from each other. The repulsive force cannot be attributed solely to the fluctuations since the fluctuations in the inner region are smaller than in the outside region when $R$ is small. We therefore need another force to explain why the two spheres separate. What we propose is that differences in the mean speed should also be considered (the fluctuations are much smaller than the mean velocities for frequencies above $\left.f_{\text {th }}\right)$. We suppose that along a streamline $P+\rho U^{2}=$ const ( $P$ is the pressure, $\rho$ is the density, and $U$ is the mean velocity) just like for irrotational fluids (Bernoulli's equation). An increase in $U$ reduces the pressure $P$. Now, and because the velocity is smaller in the inside region than in the outside, the pressure is higher in the inside region. We assume that the constant is the same for a streamline in the region between the particles and for a streamline in the outside region which amounts to assuming that the total pressure is the same along the line where the flow of the glass beads intersects the cylinder wall. The pressure difference between the two regions is given by $\rho U^{2}$ (out) $\rho U^{2}$ (in) therefore giving a repulsive force. The assumptions leading to this estimate of the pressure are not justified a priori; however, rapid shallow granular flows may be described by hydrodynamiclike expressions where Bernoulli's law applies [18]. Here, we can only justify this a posteriori. Therefore, the repulsive force we propose is proportional to the difference in the dynamic pressure $\rho\left[U_{\text {mean }}^{2}(\right.$ out $\left.)-U_{\text {mean }}^{2}(R)\right]$. The total force should then be proportional to $\delta U_{r}^{2}=\left[U_{\text {mean }}^{2}(\right.$ out $\left.)-U_{\text {mean }}^{2}(R)\right]+\delta U_{a}^{2}(R)$. The results of our measurements are shown in Fig. 4(d) which shows that $\delta U_{r}^{2}$ is positive and therefore consistent with a repulsion. The decrease versus $R$ is also consistent with the assumed variation versus $R$ as the continuous line indicates. The reasons why $\left[U_{\text {mean }}^{2}(\right.$ out $\left.)-U_{\text {mean }}^{2}(R)\right]$ are not present for the attraction are not very clear to us at 


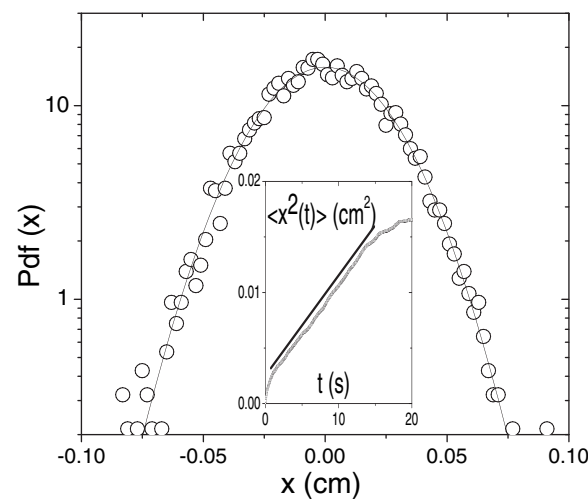

FIG. 5. The PDF of the position fluctuations of a single steel sphere with a Gaussian fit $(f=2 \mathrm{~Hz})$. Inset: the mean squared displacement of the sphere vs time $(f=3.3 \mathrm{~Hz})$.

present. However, we believe that the smallness of $U_{\text {mean }}$ for $f<f_{\text {th }}$ with respect to the fluctuations is the main reason. Indeed, our measurements show that at low frequencies, the fluctuations dominate the velocity field and $U_{\text {rms }}$ is at least twice $U_{\text {mean }}$. At higher frequencies $\left(f>f_{\text {th }}\right)$ the mean velocity becomes greater than $U_{\text {rms }}$ and the regime is mostly dominated by the mean velocity.

The value of $A$ can be extracted from these forces as $A=$ $\frac{1}{m} \frac{1}{4} \delta P(R) S R=\frac{1}{2 m} \frac{1}{4} \rho \delta U_{r, a}^{2}(R) S R$ where $\rho$ is the density of the glass beads, $m$ is the mass of the spheres, and $S$ is their surface area. The term proportional to the separation velocity $v$ invokes the equivalent of a mobility for the granular material. In order to estimate the value of $B$ we have done measurements on single spheres. By tracking the movement of the sphere, we noted large fluctuations in its axial position. From the position fluctuations versus time, we extracted a diffusion constant $D$ for the different frequencies examined here. A typical PDF of the position fluctuations of the single spheres and a typical mean squared displacement is shown in Fig. 5. The PDF comes out to be Gaussian. The mean squared displacement is linear versus time so $D$ can be measured. $D$ increases for increasing frequency. In order to extract the value of $B$, we assume that $D$ is given by a Stokes-Einstein type of expression. Here we used the velocity fluctuations to estimate the granular temperature as explained above. This gives a value for $B=\frac{1}{2} m_{g} U_{\text {rms }}^{2} / m D\left(m_{g}\right.$ is the mass of the glass beads and $U_{\text {rms }}$ is the rms value of the velocity fluctuations of the glass beads). Since the two spheres drift apart from each other to a well-defined separation $R_{\max }, C=$ $-A / R_{\max }$. The maximal separation is fixed by the ratio of $A$ and $C$.

The value of $B$, calculated from the measured diffusion constant, is roughly $30 \mathrm{~s}^{-1}$ and is weakly dependent on the frequency. Fixing this parameter, the values of $A$ and $C$ are obtained from fitting the separation $R(t)$ in Figs. 4(a) and 4(b). The value of $A$ comes out to be $20 \mathrm{~cm}^{2} / \mathrm{s}^{2}$ for the separation and $-8.5 \mathrm{~cm}^{2} / \mathrm{s}^{2}$ for the approach while $C$ is -5 and $3.7 \mathrm{~cm} / \mathrm{s}^{2}$ for the separation and the approach, respectively. A typical value of $A$ deduced from the pres- sure measurements is $20.5 \mathrm{~cm}^{2} / \mathrm{s}^{2}$ for the separation and $-2.5 \mathrm{~cm}^{2} / \mathrm{s}^{2}$ for the approach. These values, although deduced from a totally different measurement, come out to be of the same order of magnitude as the values deduced from fitting the trajectories versus time. The value of $C$ turns out to be smaller than the drag measured in $[16,17]$; however, the experimental conditions are different in the sense that the spheres here move in a fluidized medium and perpendicularly to the motion of the small grains. The value of $C$ is also smaller than typical dynamic friction coefficients and here also the fact that the medium is fluidized and the fact that the spheres may roll on top of the small grains may be possible reasons for such a difference.

We have presented a study of segregation in an assembly of grains composed of very small and very large particles in a rotating cylindrical cell. We have observed that the large spheres have a tendency to aggregate below a welldefined threshold frequency or to separate above this threshold giving rise to an ordered structure. The transition from aggregation to well-separated particles seems gradual. The effects of the velocity fluctuations of the small beads seem to be the main agents in inducing the observed interactions between the large particles. This is a new mechanism for segregation or mixing. Other features of the experiments and notably the repulsive force observed call for more theoretical understanding.

[1] H. M. Jaeger, S. R. Nagel, and R. P. Behringer, Rev. Mod. Phys. 68, 1259 (1996).

[2] A. Kudrolli, Rep. Prog. Phys. 67, 209 (2004).

[3] T. Shinbrot and F. J. Muzzio, Nature (London) 410, 251 (2001).

[4] T. Mullin, Science 295, 1851 (2002).

[5] Y. Oyama, Bull. Inst. Phys. Chem. Res. (Tokyo), Rep. 18, 600 (1939).

[6] K. M. Hill, A. Caprihan, and J. Kakalios, Phys. Rev. Lett. 78, 50 (1997).

[7] K. Choo et al., Phys. Rev. E 58, 6115 (1998).

[8] O. Zik et al., Phys. Rev. Lett. 73, 644 (1994).

[9] D. C. Rapaport, Phys. Rev. E 65, 061306 (2002).

[10] P. M. Reis and T. Mullin, Phys. Rev. Lett. 89, 244301 (2002).

[11] M. Bose, P. R. Nott, and V. Kumaran, Europhys. Lett. 68, 508 (2004).

[12] D. A. Huerta and J. C. Ruiz-Suarez, Phys. Rev. Lett. 92, 114301 (2004).

[13] P. K. Haff, J. Fluid Mech. 134, 401 (1983).

[14] E. L. Grossman, T. Zhou, and E. Ben-Naim, Phys. Rev. E 55, 4200 (1997).

[15] R. Albert et al., Phys. Rev. Lett. 82, 205 (1999).

[16] D. Chehata, R. Zenit, and C. R. Wassgren, Phys. Fluids 15, 1622 (2003).

[17] J. Geng and R. P. Behringer, Phys. Rev. Lett. 93, 238002 (2004).

[18] J. M. N. T. Gray, Y.-C. Tai, and S. Noelle, J. Fluid Mech. 491, 161 (2003). 